“( 2015 IEEE. Personal use of this material is permitted. Permission from IEEE must be obtained for all other uses, in any current or future media, including reprinting/republishing this material for advertising or promotional purposes, creating new collective works, for resale or redistribution to servers or lists, or reuse of any copyrighted component of this work in other works." 


\title{
An Extended Kalman Filter for Localisation in Occupancy Grid maps
}

\author{
Lakshitha Dantanarayana ${ }^{\ddagger}$, Gamini Dissanayake ${ }^{\ddagger}$, Ravindra Ranasinghe ${ }^{\ddagger}$ and Tomonari Furukawa ${ }^{\ddagger}$ \\ †Centre for Autonomous Systems, \\ University of Technology, Sydney, NSW, Australia. \\ Email:\{Lakshitha.Dantanarayana, Gamini.Dissanayake, Ravindra.Ranasinghe\}@uts.edu.au \\ $\dagger$ Dept. of Mechanical Engineering, \\ Virginia Tech, Blacksburg, VA, USA. \\ Email: tomonari@vt.edu
}

\begin{abstract}
The main contribution of this paper is an extended Kalman filter (EKF) based framework for mobile robot localisation in occupancy grid maps (OGMs), when the initial location is approximately known. We propose that the observation equation be formulated using the unsigned distance transform based Chamfer Distance (CD) that corresponds to a laser scan placed within the OGM, as a constraint. This formulation provides an alternative to the ray-casting model, which generally limited localisation in OGMs to Particle Filter (PF) based frameworks that can efficiently deal with observation models that are not analytic. Usage of an EKF is attractive due to its computational efficiency, especially as it can be applied to modern day field robots with limited on-board computing power. Furthermore, well-developed tools for dealing with potential outliers in the observations or changes to the motion model, exists in the EKF framework. The effectiveness of the proposed algorithm is demonstrated using a number of simulation and real life examples, including one in a dynamic environment populated with people.
\end{abstract}

\section{INTRODUCTION}

The problem of localising a mobile robot within a given map in an indoor environment using sensors mounted on it has been a problem studied for over two decades. Extended Kalman filter (EKF) based algorithms can efficiently estimate the robot pose by fusing robot odometry information and range-bearing sensor observations that corresponds to these geometric primitives [1]. To date, it is only possible to use an EKF for localisation if the map of the environment consists of a collection of geometric primitives such as points, lines or splines. These localisers require a sensor processing step where lines or points are first extracted from the measurements. The observations that do not belong to these geometric primitives are then discarded, resulting in a significant information loss that may be detrimental in environments sparsely populated with features. Recently EKF based localisation algorithms were heavily used even on small humanoid platforms where computational power is highly limited [2], [3], [4]. EKFs have also been successfully used to solve a large number of problems in robot navigation including the more challenging Simultaneous Localisation and Mapping (SLAM) problem [5].

However if the environment map is available in the form of an occupancy grid map (OGM), particle filter (PF) based approaches [6] have been the preferred method for robot localisation due to their ability to exploit all the measurements available in a range scan. Furthermore, they are relatively easy to implement, and are capable of global localisation; ability to deal with the situation when a suitable initial estimate for the robot pose is unavailable. The widely used Adaptive MonteCarlo localisation (AMCL) [7], [8], [9], [10], [11], [12], that is also available as a part of the popular Robot Operating System (ROS) [13] is a PF based probabilistic approach for localisation

The PF based approaches use a sensor model and a set of particles distributed around hypothesised robot locations to estimate the true pose of the robot. A sufficiently large number of particles, adequate to describe the probability density function describing the robot pose needs to be selected in order to generate location estimates with acceptable accuracy. This is the main drawback of this algorithm as the computational efficiency of the PF is directly related to the number of particles used for the computation. In AMCL [10], there are many tuning parameters and strategies to dynamically manage the number of particles at an optimum level. Within the PF framework, it is also not straightforward to identify outliers or dynamic objects. In addressing this problem AMCL uses a "model" which categorises the range readings by analysing the probable causes of such outliers and penalising these observations during the particle update step. However, [10] cautions that this method would only work in certain limited situations and the categories should be analysed according to the environment.

Therefore, while a PF is indispensable in localising a mobile robot when its initial position is unknown (Kidnapped Robot Problem), it could be argued that once the approximate location is known and if the length of time over which odometry is used to predict robot pose without external sensor data is small (as is typically the case with modern high scan rate laser range finders), powerful machinery embedded in the PF for non-Gaussian estimation is not essential.

In this paper we propose, for the first time to our knowledge, an EKF based algorithm to localise a mobile robot with a laser range finder in a two dimensional OGM when the initial location is approximately known. The key challenge has been to formulate an appropriate measurement equation that can 
be used to predict the expected observations from a range finder. In the PF framework, this is done through ray-casting. However this strategy is not suitable for an EKF as an efficient implementation requires the Jacobians of the observation function in closed form. Therefore we propose that the observation equation be formulated using the unsigned distance transform based Chamfer Distance (CD) that corresponds to a laser scan placed within the OGM as a constraint. However, such a constraint relates the robot state and the range readings in an implicit function and as such is not in a form suitable for a standard implementation of the EKF. The strategy proposed by Steffen et. al. [14] is, therefore, used to implement the EKF. In contrast to typical particle filter implementations, the algorithm proposed in this paper is easy to tune, as only the parameters corresponding to the noise values for the inputs to motion and measurement models are required. Furthermore, it is relatively easy to deal with outliers present through a probabilistic strategy that only accepts measurements subjected to a desired confidence level.

This paper is structured as follows: Sect. II describes the formulation of an EKF based localiser using unsigned distance transform based $\mathrm{CD}$ to form an implicit constraint. It also discusses a method that can be used to deal with the outliers that are almost always present in the observations captured in practical scenarios. Sect. III demonstrate the effectiveness of the proposed algorithm using a number of simulation and real life examples, including one in a dynamic environment populated with people. A discussion of the results and a conclusion is presented in the Sect. IV \& V.

\section{Formulation of the Extended Kalman Filter}

\section{A. Distance Transform Based Observation Model}

The distance transform is an implicit shape representation popular within the computer vision community. For an environment represented using a binary occupancy grid, distance transform corresponding to each grid cell indicates the minimum distance from that point to the closest occupied cell. Distance transform based CD [15] is a measure of the misalignment between two binary images, where CD is zero when two images are perfectly aligned. This property forms the basis of the observation model described in this section.

For a given OGM with the set of occupied pixels $V$, the distance transform at the location $\mathbf{x}$, can be generated using an unsigned distance function via (1), which specifies the Euclidean distance from that pixel to the nearest edge pixel $v_{j}$ in $V$ [16].

$$
D T(\mathbf{x})=\min _{v_{j} \in V}\left|\mathbf{x}-\mathbf{v}_{j}\right|
$$

While the distance between any point in the environment and the nearest object is a continuous value, the distance transform as described above quantises these distances into cell numbers. Furthermore, derivatives of the distance transform are not continuous at points which belong to the map or to the cut-locus [17]. As the purpose of the exercise is to use the distance transform based $\mathrm{CD}$ as the basis for an observation

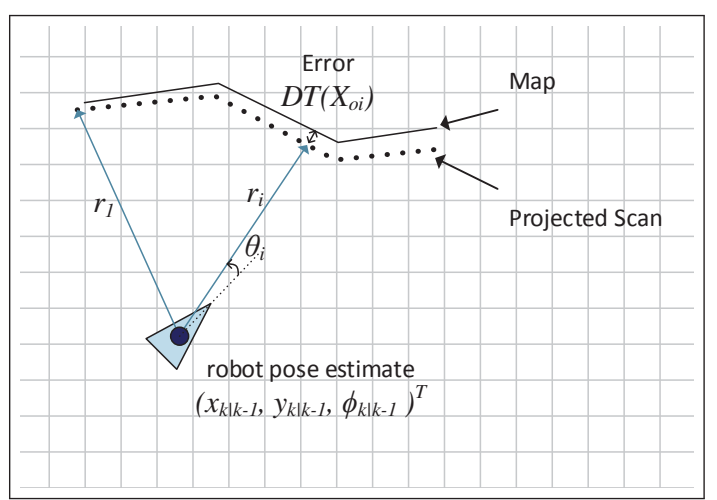

Fig. 1: Projection of the laser scan from the a priori robot pose.

model within an EKF framework, a cubic spline approximation based interpolation algorithm is used to compute the distance transform and its derivatives at any given location in the map. Future references to DT in this paper refer to the interpolated version of the DT matrix. These values are precomputed and stored so that the computational effort required during runtime is minimised.

When the robot equipped with a range scanner is located at pose $X_{k \mid k-1}=\left(x_{k \mid k-1}, y_{k \mid k-1}, \phi_{k \mid k-1}\right)^{\top}$, (Fig. 1), the observation vector $\mathbf{z}$ consists of $n$ range readings $(r)$ at given bearings $\left(\theta_{i}\right)$. It is proposed that the $\mathrm{CD}$ between the map of the environment and the Cartesian coordinates $\mathbf{X}_{o}$ that correspond to the range measurement endpoint shown in(2)

$$
h(\mathbf{X}, \mathbf{z})=\frac{1}{n} \sum_{i=0}^{n-1} D T\left(X_{o_{i}}\right)=C D
$$

where $\mathbf{X}_{o}$ is given by,

$$
\mathbf{X}_{o_{i}}=\left\{\begin{array}{l}
x_{o_{i}} \\
y_{o_{i}}
\end{array}\right\}=\left\{\begin{array}{l}
x_{k \mid k-1}+r_{i} \cos \left(\theta_{i}+\phi_{k \mid k-1}\right) \\
y_{k \mid k-1}+r_{i} \sin \left(\theta_{i}+\phi_{k \mid k-1}\right)
\end{array}\right\}
$$

The CD of a single range scan, as the estimate for the position of the robot $(x, y)$ is varied in the vicinity of the true pose (at $\approx 1.1,1.1$ for Fig. 2.a and $\approx 0.45,0.45$ for Fig. 2.b) is shown in the contour map in Fig. 2 [18]. The minimum CD, which will be equal to zero when there is no measurement noise, is obtained when the robot is placed at its true pose resulting in the map and the laser scan to be fully aligned. Therefore, setting CD to zero in (2) yields the measurement equation (4), suitable for robot localisation.

$$
h(\mathbf{X}, \mathbf{z})=0
$$

Traditional formulation of the EKF requires an observation equation of the form $z=h(x)$. The alternative formulation that is proposed below can directly deal with an implicit form of the measurement equation, is an adaptation of [14].

\section{B. Prediction}

Let the estimate of the robot pose be $X_{k-1 \mid k-1}=$ $\left(x_{k-1 \mid k-1}, y_{k-1 \mid k-1}, \phi_{k-1 \mid k-1}\right)^{\top}$ and is subjected to a control 

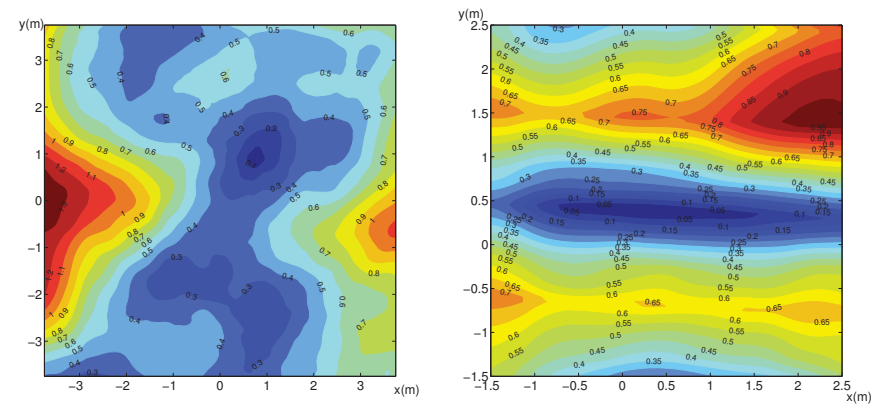

Fig. 2: CD variation in the vicinity of the true robot pose for two parts of the Intel Research Labs dataset.[18]

command of $u_{k}=\left(v_{k}, \omega_{k}\right)^{\top}$, where $v_{k}$ is the linear velocity and $\omega_{k}$ is the angular velocity demand over a period of $\Delta t$.

Then the predicted location of the robot is given by (5) and it's covariance by,

$$
\begin{gathered}
X_{k \mid k-1}=F\left(X_{k-1 \mid k-1}, u_{k} \cdot \Delta t\right) \\
P_{k \mid k-1}=\nabla F_{\mathbf{X}} P_{k-1 \mid k-1} \nabla F_{\mathbf{x}}^{\top}+\nabla F_{u} Q_{k} \nabla F_{u}^{\top}
\end{gathered}
$$

where $\nabla F_{\mathbf{X}}$ and $\nabla F_{\mathbf{u}}$ are respectively the Jacobin of the control function $F$ w.r.t. $X$ and $u$, obtained by linearising about the robot pose estimate $X_{k-1 \mid k-1}$, while $Q_{k}$ is the control noise covariance matrix.

\section{Observation}

$$
h(\mathbf{X}, \mathbf{z})=0
$$

Assuming that each range measurement of the scan $\mathbf{z}$ corrupted by noise $\eta_{r}$ with $\mathscr{N}\left(0, \sigma_{r}^{2}\right)$ and bearing $\theta$ is noise free, the covariance of the measurement vector is given by the diagonal matrix, $\Sigma_{\mathbf{z}}=\operatorname{diag}\left(\sigma_{r}^{2}\right)_{n \times n}$.

\section{Update}

Update equations can be written as follows based on the derivations presented in [14]. The filter gain $K$ is given by,

$$
K=P_{k \mid k-1} \nabla h_{\mathrm{x}}^{\top}\left(\nabla h_{\mathrm{x}} P_{k \mid k-1} \nabla h_{\mathrm{x}}^{\top}+\nabla h_{\mathrm{z}}^{\top} \Sigma_{\mathrm{z}} \nabla h_{\mathrm{z}}\right)^{-1}
$$

The state update is given by,

$$
X_{k \mid k}=X_{k \mid k-1}+K\left(-h\left(\mathbf{X}_{k \mid k-1}, \mathbf{z}\right)\right)
$$

while the covariance update is,

$$
P_{k \mid k}=\left(I-K \nabla h_{\mathrm{x}}\right) P_{k \mid k-1}
$$

The Jacobians $\nabla h_{\mathrm{x}}$ and $\nabla h_{\mathrm{z}}$ (defined in (10)) at the appropriate linearisation points can be calculated using (11).

$$
\begin{gathered}
\nabla h_{\mathbf{x}}=\left.\frac{\partial h(\mathbf{X}, \mathbf{z})}{\partial \mathbf{X}}\right|_{\mathbf{z}, \mathbf{X}_{k \mid k-1}} \\
\nabla h_{z}=\left.\frac{\partial h(\mathbf{X}, \mathbf{z})}{\partial \mathbf{z}}\right|_{\mathbf{z}, \mathbf{X}_{k \mid k-1}} \\
\left.\frac{\partial h(\mathbf{X}, \mathbf{z})}{\partial x}\right|_{\mathbf{z}, \mathbf{X}_{k \mid k-1}}=\frac{\partial D T}{\partial x_{o_{i}}} \cdot \frac{\partial x_{o_{i}}}{\partial x} \\
\left.\frac{\partial h(\mathbf{X}, \mathbf{z})}{\partial y}\right|_{\mathbf{z}, \mathbf{X}_{k \mid k-1}}=\frac{\partial D T}{\partial y_{o_{i}}} \cdot \frac{\partial y_{o_{i}}}{\partial y} \\
\left.\frac{\partial h(\mathbf{X}, \mathbf{z})}{\partial \phi}\right|_{\mathbf{z}, \mathbf{X}_{k \mid k-1}}=\frac{\partial D T}{\partial x_{o_{i}}} \cdot \frac{\partial x_{o_{i}}}{\partial \phi}+\frac{\partial D T}{\partial y_{o_{i}}} \cdot \frac{\partial y_{o_{i}}}{\partial \phi} \\
\left.\frac{\partial h(\mathbf{X}, \mathbf{z})}{\partial r_{i}}\right|_{\mathbf{z}, \mathbf{X}_{k \mid k-1}}=\frac{\partial D T}{\partial x_{o_{i}}} \cdot \frac{\partial x_{o_{i}}}{\partial r_{i}}+\frac{\partial D T}{\partial y_{o_{i}}} \cdot \frac{\partial y_{o_{i}}}{\partial r_{i}}
\end{gathered}
$$

$\frac{\partial D T}{\partial x_{o_{i}}}$ and $\frac{\partial D T}{\partial y_{o_{i}}}$ in (11) can be obtained by looking up the gradients of the distance transform with respect to global $x_{o}, y_{o}$ coordinates. As previously mentioned, the distance transform and its derivatives can be precomputed using the grid map and stored to make the gradient calculations computationally efficient. The remaining components of the gradient can be analytically derived from (3).

\section{E. Improving the Robustness of the Algorithm}

A simple innovation gate is used in the algorithm to filter outliers that are related to objects not present in the map, such as people walking around. The individual entries of $D T\left(\mathbf{X}_{o}\right)$ vector is tested to be within $2 \sigma_{\mathrm{DT}}$ bounds and the ones that are beyond these bounds are ignored.

\section{EXPERIMENTS}

In order to evaluate different aspects of performance of the proposed algorithm, three experiments are presented in this section, conducted using three different datasets: a simulation based data set and two real world datasets.

The simulation based dataset (Dataset 1) was generated using Player/Stage [19] robot simulator available in ROS, and the example map "Hospital Section" which comes with ROS. This dataset consists of the ground truth and exact sensor uncertainties. The sensor readings were collected at a $10 \mathrm{~Hz}$ frequency and the noise parameters shown in Table I were used during data collection.

TABLE I: Noise parameters for Dataset 1

\begin{tabular}{|l|l|}
\hline Parameter & Noise Value \\
\hline Laser range finder Measurement Noise & $\mathscr{N}\left(0,0.02^{2} \mathrm{~m}^{2}\right)$ \\
Linear velocity noise & $\mathscr{N}\left(0,0.04^{2} \mathrm{~m}^{2} \mathrm{~s}^{-2}\right)$ \\
Angular velocity noise & $\mathscr{N}\left(0,0.01^{2} \mathrm{rad}^{2} \mathrm{~s}^{-2}\right)$ \\
\hline
\end{tabular}


Fig. 3 shows the estimated poses and the map drawn from the laser scans projected from these estimated poses. The $2 \sigma$ uncertainty ellipses are also depicted (plotted intermittently to avoid clutter) at the relevant poses. As the ground truth is available, the error of each of the components in the pose, $x, y, \phi$ are shown Fig. 4 with the respective $2 \sigma$ bounds, clearly demonstrating that the EKF produces consistent pose estimates.

In order to evaluate the algorithm's performance in a real environment with true noise characteristics, the second experiment was conducted with the popular the Intel Research Lab [20] (Dataset 2) public domain dataset. The OGM was created with the aid of the GMapping [21] algorithm by selectively choosing every other laser scan. Remaining laser scans were used to evaluate the proposed method of localisation. As with the previous dataset the estimated pose trail and the laser projection from those estimated poses are represented in the Fig. 5 with the uncertainty ellipses.

Finally, another real world dataset collected at Centre for Autonomous Systems(CAS), University of Technology, Sydney (Dataset 3) was used for evaluating the proposed algorithm. Scans in this dataset consist of the three people moving about the area as the robot moves so that the impact of unknown dynamic objects can be examined. It was collected using a TurtleBot ${ }^{\mathrm{TM}}$ from WillowGarage ${ }^{\mathrm{TM}}$ and Clearpath Robotics $^{\mathrm{TM}}($ Fig. 6) with a Hokuyo® UTM-30LX, 30m laser range finder, traversing the environment in multiple loops. The first run, conducted when the environment is free of dynamic objects was used to create the OGM using GMapping. The proposed technique was then used to localise the robot within this map during subsequent loops. Fig. 7 shows the poses and laser range readings superimposed on each other based on the estimated robot pose. It can be seen that the walls in the environment are aligned well, providing a qualitative indication of the accuracy of the pose estimates. The clutter seen in the corridors are a result of people moving around in the environment and occluding the laser.

The Experiments were conducted on Mathworks ${ }^{\circledR}$ MATLAB 2013b environment using single threaded programming on a single core of Intel® Core ${ }^{\mathrm{TM}}$ i5-2400 CPU @ 3.10GHz.

\section{Discussion}

When observing results of all three datasets it can be seen that laser range finder scans are considerably well matched with the map walls providing a qualitative indication of the pose estimation accuracy. The pose uncertainty ellipses also show obvious characteristics that are to be expected, for example, elongated ellipses confirming that the uncertainty of the pose estimate along the direction of the corridor is larger due to the lack of information available in this direction.

The error plots for Dataset 1 shown in Fig. 4 confirms that the pose error for each component in the state vector is more than $95 \%$ within the $2 \sigma$ bounds and therefore the estimate is consistent throughout.

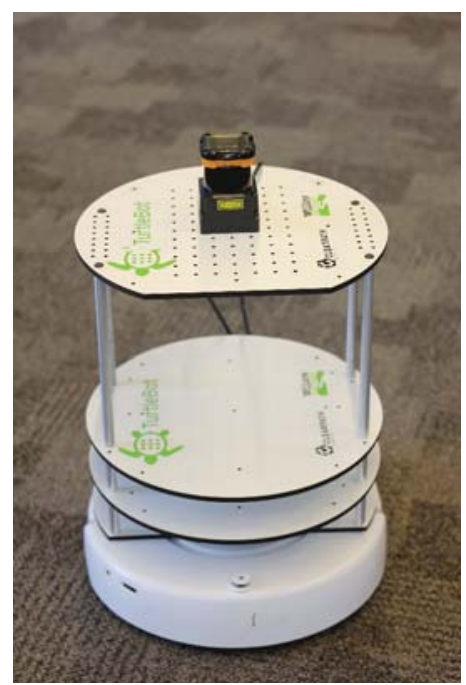

Fig. 6: TurtleBot ${ }^{\mathrm{TM}}$ from WillowGarage ${ }^{\mathrm{TM}}$ and Clearpath Robotics $^{\mathrm{TM}}$. A Hokuyo ${ }^{\circledR}$ laser range finder is mounted on top of the robot.

The results from Dataset 2 shown in Fig. 5 the walls confirms that the proposed algorithm can cope up with real world noise characteristics.

From the experiments with Dataset 3 , it can be seen that even amidst clutter the proposed algorithm can effectively reject outliers in the environment. Furthermore, as before the laser scans successfully align with the wall suggesting accurate localisation. It was further observed that a single update of the EKF takes less than $15 \mathrm{~ms}$ in average in the MATLAB environment.

\section{CONCLUSION}

In this paper, the problem of localising a mobile robot on an OGM of an indoor 2D environment with the use of a laser range finder sensor mounted on the robot is formulated within an EKF framework. The observation equation for the EKF is based on the implicit constraint that the unsigned distance transform based CD of the range scan should equal to zero.

It is also shown that the algorithm makes use of precomputed distance transform values and their gradients, which makes the algorithm computationally efficient. A method for making the filter more robust against outliers in the measurement as a result of dynamic objects in the environment is also proposed.

The performance of the algorithm has been experimentally verified using a Player/Stage based simulation, the Intel Research labs public domain dataset and a dataset collected by the authors using a TurtleBot ${ }^{\mathrm{TM}}$ robot from Willow Garage ${ }^{\mathrm{TM}}$ and Clearpath Robotics ${ }^{\mathrm{TM}}$ in a dynamic environment at the Centre for Autonomous Systems, University of Technology, Sydney. Results showing the well alignment of the laser scans drawn from the estimated poses with the wall suggests accurate localisation, and the error plots show that the EKF is consistent. 


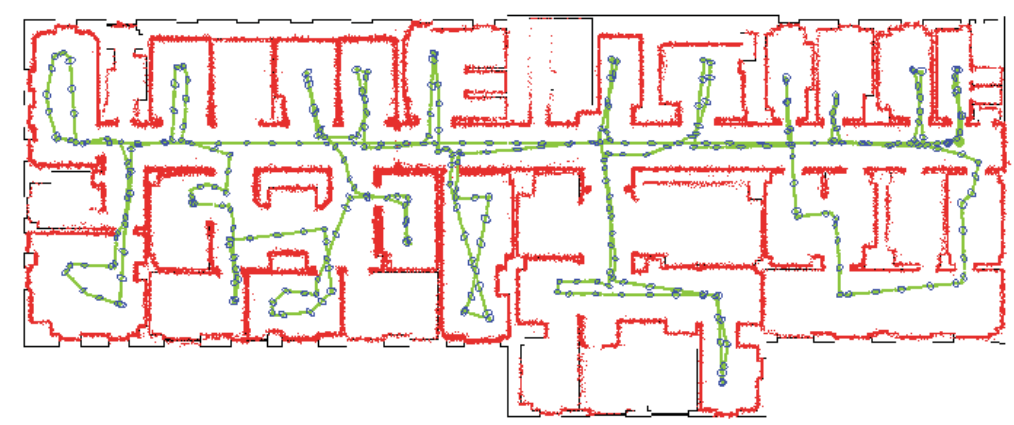

Fig. 3: Simulation dataset. Poses, laser trace and $2 \sigma$ uncertainty ellipses are shown.
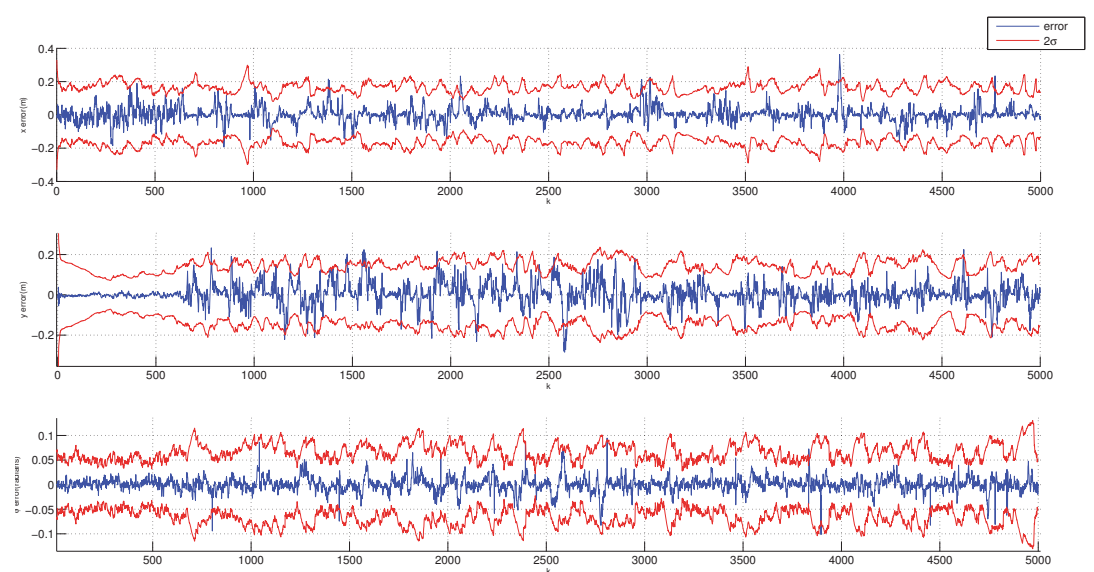

Fig. 4: Plots of errors against ground truth with their $2 \sigma$ bounds.

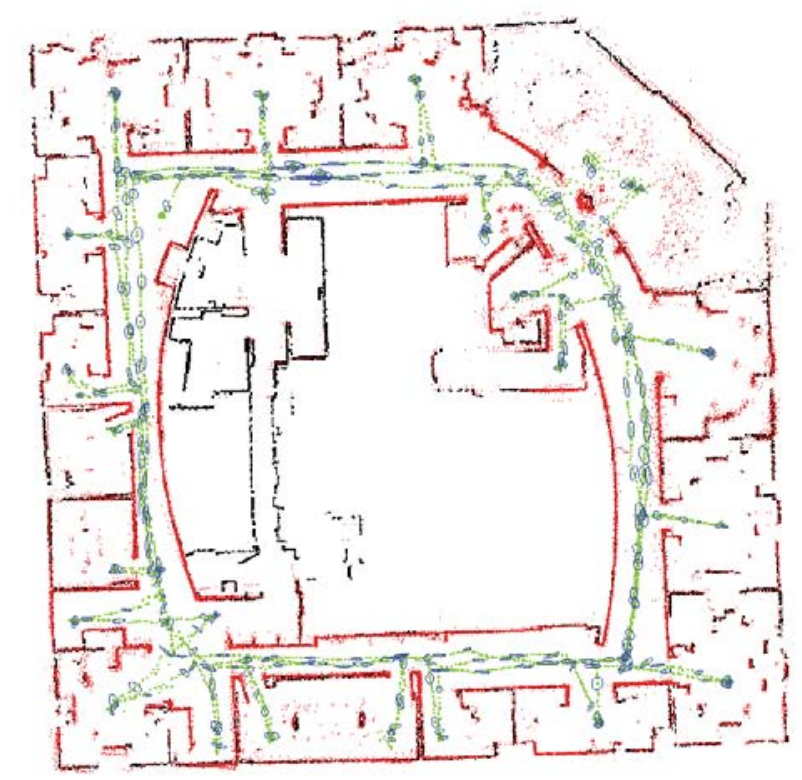

Fig. 5: Intel Dataset. Poses, laser trace and $2 \sigma$ uncertainty ellipses are shown. 


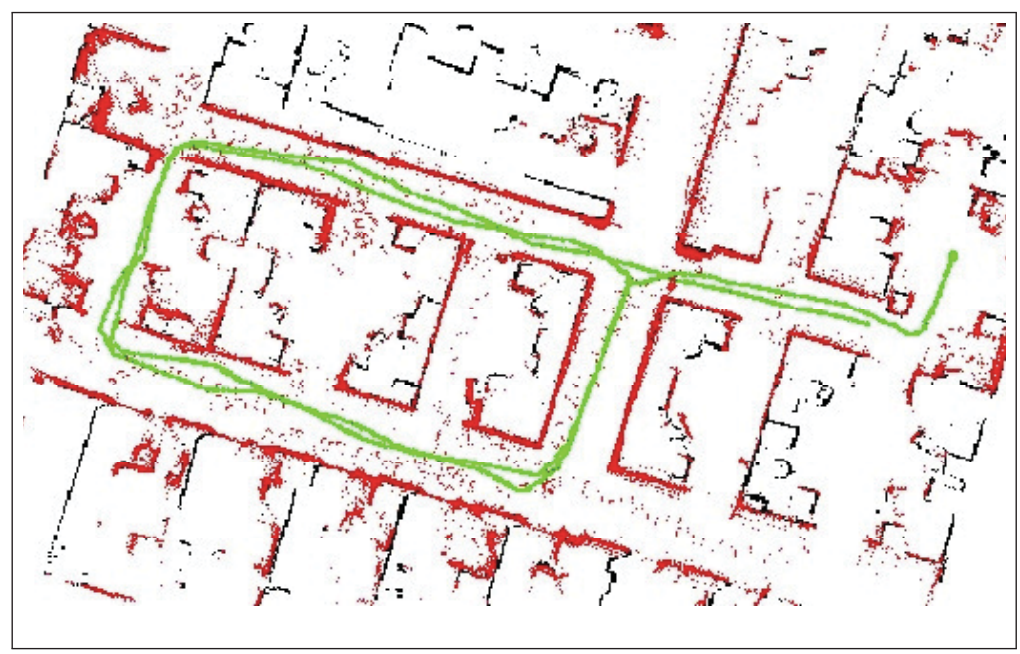

Fig. 7: CAS Dataset. Poses and laser trace. Clutter in corridors represents footsteps of people moving about.

There are three different versions of distance transform based observation models that can be used with the proposed algorithm. Namely, signed distance transform based model, unsigned distance transform based model and the Chamfer Distance based model that is presented in this paper. Further investigation to assess the technical merits of the proposed distance based observation model under each of these representations is planned for future work.

Further online experiments of the algorithm is planned with a $\mathrm{C}++$ / ROS based implementation in the immediate future. A proper benchmarking of the computational performance of the filter would also be possible with such an implementation. As the proposed algorithm is much more lightweight and computationally efficient than using a PF based localisation method, authors also plan to use and evaluate it with the next version Smart Hoist system [22] to aid its navigation capabilities.

\section{REFERENCES}

[1] J. Leonard and H. Durrant-Whyte, "Mobile robot localization by tracking geometric beacons," IEEE Transactions on Robotics and Automation, vol. 7, pp. 376-382, June 1991. 1

[2] M. J. Quinlan and R. H. Middleton, "Multiple model Kalman filters: A localization technique for RoboCup soccer," in Lecture Notes in Computer Science (including subseries Lecture Notes in Artificial Intelligence and Lecture Notes in Bioinformatics), vol. 5949 LNAI, pp. 276-287, 2010. 1

[3] G. Jochmann, S. Kerner, S. Tasse, and O. Urbann, "Efficient multihypotheses unscented kalman filtering for robust localization," in Lecture Notes in Computer Science (including subseries Lecture Notes in Artificial Intelligence and Lecture Notes in Bioinformatics), vol. 7416 LNCS, pp. 222-233, 2012. 1

[4] S. Tasse, M. Hofmann, and O. Urbann, "On Sensor Model Design Choices for Humanoid Robot Localization," in RoboCup 2012: Robot Soccer World Cup XVI, pp. 380-390, 2013. 1

[5] G. Dissanayake, P. Newman, S. Clark, H. F. Durrant-Whyte, and M. Csorba, "A solution to the simultaneous localization and map building (SLAM) problem," IEEE Transactions on Robotics and Automation, vol. 17, pp. 229-241, June 2001. 1

[6] C. Stachniss and W. Burgard, "Particle Filters for Robot Navigation," Foundations and trends in Robotics, vol. 3, no. 4, pp. 211-282, 2014 1
[7] F. Dellaert, D. Fox, W. Burgard, and S. Thrun, "Monte Carlo localization for mobile robots," Proceedings 1999 IEEE International Conference on Robotics and Automation (Cat. No.99CH36288C), vol. 2, 1999. 1

[8] S. Thrun, D. Fox, W. Burgard, and F. Dellaert, "Robust Monte Carlo localization for mobile robots," Artificial Intelligence, vol. 128, pp. 99 141, May 2001. 1

[9] S. Thrun, "A Probabilistic On-Line Mapping Algorithm for Teams of Mobile Robots," May 2001. 1

[10] S. Thrun, W. Burgard, and D. Fox, Probabilistic robotics. The MIT Press, 2005. 1

[11] D. Fox, "Adapting the Sample Size in Particle Filters Through KLDSampling," The International Journal of Robotics Research, vol. 22, pp. 985-1003, Dec. 2003. 1

[12] S. S. Srinivasa, D. Ferguson, C. J. Helfrich, D. Berenson, A. Collet, R. Diankov, G. Gallagher, G. Hollinger, J. Kuffner, and M. V. Weghe, "HERB: A home exploring robotic butler," Autonomous Robots, vol. 28, pp. 5-20, Nov. 2010. 1

[13] WillowGarage, "Robot Operating System(ROS)," in http://wiki.ros.org, 2007. 1

[14] R. Steffen, "A Robust Iterative Kalman Filter Based On Implicit Measurement Equations," Photogrammetrie - Fernerkundung - Geoinformation, vol. 2013, pp. 323-332, Aug. 2008. 2, 3

[15] H. Barrow, J. Tenenbaum, R. Bolles, and H. Wolf, "Parametric correspondence and chamfer matching: Two new techniques for image matching," in 5th international joint conference on Articial intelligence - Volume 2, (San Francisco, CA, USA), pp. 659-663, Morgan Kaufmann Publishers Inc., 1977. 2

[16] M.-Y. Liu, O. Tuzel, A. Veeraraghavan, and R. Chellappa, "Fast directional chamfer matching," in 2010 IEEE Computer Society Conference on Computer Vision and Pattern Recognition, pp. 1696-1703, IEEE, June 2010. 2

[17] M. W. Jones, J. A. Bæ rentzen, and M. Sramek, "3D distance fields: A survey of techniques and applications," IEEE Transactions on Visualization and Computer Graphics, vol. 12, pp. 581-599, Jan. 2006. 2

[18] L. Dantanarayana, R. Ranasinghe, and G. Dissanayake, "C-LOG: A Chamfer Distance based method for localisation in occupancy gridmaps," in 2013 IEEE/RSJ International Conference on Intelligent Robots and Systems, pp. 376-381, IEEE, Nov. 2013. 2, 3

[19] R. Vaughan, "Player Project,Stage robot simulator for ROS," in http://wiki.ros.org/stage, 2009. 3

[20] D. Hähnel, "Intel Research Lab (Seattle) Dataset," 2000. 4

[21] G. Grisetti, C. Stachniss, and W. Burgard, "Improved techniques for grid mapping with Rao-Blackwellized particle filters," IEEE Transactions on Robotics, vol. 23, pp. 34-46, Feb. 2007. 4

[22] R. Ranasinghe, L. Dantanarayana, A. Tran, S. Lie, M. Behrens, and L. Liu, "Smart Hoist: An Assistive Robot to Aid Carers," in 13th International Conference on Control, Automation, Robotics and Vi sion(ICARCV), 2014. 6 